\title{
OPTION PRICING IN A REGIME-SWITCHING MODEL USING THE FAST FOURIER TRANSFORM
}

\author{
R. H. LIU, Q. ZHANG, AND G. YIN \\ Received 6 December 2005; Revised 11 June 2006; Accepted 5 July 2006
}

This paper is concerned with fast Fourier transform (FFT) approach to option valuation, where the underlying asset price is governed by a regime-switching geometric Brownian motion. An FFT method for the regime-switching model is developed first. Aiming at reducing computational complexity, a near-optimal FFT scheme is proposed when the modulating Markov chain has a large state space. To test the FFT method, a novel semiMonte Carlo simulation algorithm is developed. This method takes advantage of the observation that the option value for a given sample path of the underlying Markov chain can be calculated using the Black-Scholes formula. Finally, numerical results are reported.

Copyright () 2006 R. H. Liu et al. This is an open access article distributed under the Creative Commons Attribution License, which permits unrestricted use, distribution, and reproduction in any medium, provided the original work is properly cited.

\section{Introduction}

Fast Fourier transform (FFT) (see Brigham [5]) is one of the most significant advances in scientific computing; it has played an increasingly important role in financial engineering, especially for determining values of derivatives numerically. It is applicable to problems for which the characteristic functions of the underlying price processes can be obtained analytically. It has been recognized that a wide range of models considered in the literature meets this requirement. They include the stochastic volatility models, the affine jump diffusions, and the exponential Lévy models, among others; see Carr and Madan [7], Carr and Wu [8], and Duffie et al. [13] for detailed discussions of these models.

Because of its prevalence, increasing research efforts have been devoted to the FFT approach in option pricing. For example, Carr and Madan [7] illustrated the fundamental idea of using FFT for valuing European options based on the Black-Scholes setting and applied it to the variance gamma (VG) model (see Madan et al. [19]). Černý [9] presented a detailed discussion on the implementation of FFT to option pricing. Benhamou [3] used FFT for discrete Asian options valuation. Dempster and Hong [12] developed an approximation method for pricing European options on spread using the

Hindawi Publishing Corporation

Journal of Applied Mathematics and Stochastic Analysis

Volume 2006, Article ID 18109, Pages 1-22

DOI 10.1155/JAMSA/2006/18109 
two-dimensional fast Fourier transform. Lee [18] studied the error bound in using FFT for approximate valuation. Chourdakis [10] employed the fractional FFT technique (see Bailey and Swarztrauber [1]) to overcome the difficulty of the inverse (see (2.39)) between the two grid sizes in the Carr and Madan FFT approach.

Along another line, considerable attention has been focused on the regime-switching diffusion models for asset prices recently. In this setting, model parameters (rate of return, volatility, and risk-free interest rate) are assumed to depend on a finite-state Markov chain, whose states represent different "states of world" or regimes, which can describe various randomly changing economical factors. By incorporating a hidden Markov chain into the formulation, the regime-switching framework can capture the effect of those less frequent but significant events that have impact on the individual asset price behavior (especially for long-term dynamics). This is a major advantage compared with other models, see Yao1 et al. [21], and Zhang [25], among others for discussions on considerations leading to this modelling approach.

Option valuation with regime-switching has been dealt with by a host of researchers. Buffington and Elliott [6] treated both European and American options. A system of Black-Scholes like partial differential equations satisfied by the call option price is derived using risk-neutral valuation principle. For American options, they extended the approximation approach of Barone-Adesi and Whaley (see [2]) to the regime-switching setting. Bollen [4] employed lattice and simulation methods. Yaol et al. [21] studied the system of PDEs with carefully chosen smooth boundary conditions to approximate the option price and developed a successive approximation scheme. Guo [15], and Guo and Zhang [16] derived closed-form solutions for the European and perpetual American options prices, respectively, in the case when the underlying Markov chain has two states. Fuh and Wang [14] used similar ideas to study the general $m$-dimensional problem and presented an approximation method by replacing the time-dependent probability distribution of the Markov chain with its stationary distribution. Related works concerning regime-switching models include the optimal selling rules for stock trading (Guo and Zhang [17], Pemy and Zhang [20], Yin et al. [22], Zhang [25], and Zhang et al. [27]), optimal asset allocation in portfolio management (Zhang and Yin [26]), and dynamic Markowitz's asset selection problem (Zhou and Yin [28]).

Our contributions in this paper are as follows. (1) By adopting the methodology of Carr and Madan [7], we develop a fast Fourier transform approach to option pricing for regime-switching models of the underlying asset process. The Fourier transform of the option price is obtained in terms of the joint characteristic function of the sojourn times of the Markov chain. We present the joint characteristic function in explicit form for two-state $(m=2)$ Markov chains, and in terms of solutions of systems of $m$-dimensional differential equations for $m$-state case. (2) Under the formulation of a regime-switching model, to take various economical factors into consideration in modelling, the state space of the driving Markov chain is inevitably large. As a consequence, the computational complexity involved in option valuation becomes a serious issue since typically the complexity depends crucially on the state space of the Markov chain. It is thus foremost to be able to reduce the computational complexity. Here, we develop an approximation strategy for the use of FFT. The essence is multiscale modelling and near optimality. The approach 
we are taking is a two-time-scale formulation. Note that for reducing computational burden in stock liquidation, Zhang et al. [27] proposed a two-time-scale regime-switching framework based on a singular perturbation approach. The main idea is to formulate the asset model using a Markov chain with two-time-scale structure, a fast time scale and a slow one. Then the quantities associated with the fast scale can be replaced by weighted averages with respect to the corresponding stationary distributions. As a result, a limit model is obtained with reduced number of states. Solution to the limit problem provides an approximation to the original complex problem. We refer to [27] for further discussions on the two-time-scale modelling motivations for asset prices and to Yin and Zhang [23] for more illustrations on singularly perturbed Markov chains and applications. (3) As a consequence of (2), a limit price process and the associated option pricing formula are defined first. The Fourier transform of the original true option values is shown to converge to that of the limit option values as the time scale parameter $\varepsilon$ approaches zero. This result, in turn, implies the convergence of the option values by the uniqueness of the transform. Note that the state space corresponding to the jump component of the regime-switching diffusion is much smaller than that of the original system. (4) We use the FFT of the limit valuation to approximate the true option prices. Numerical results demonstrate the performance of these approximations. Most importantly, by working on the limit problem, the computational time can be reduced significantly. Noticeably, this near-optimal scheme combines the salient feature of FFT in computational speedup with that of the two-time-scale approach in structurally reducing complexity, resulting in considerable advantage over the Monte Carlo simulation and PDE methods. (5) Furthermore, we present an efficient numerical method, which is Monte Carlo simulation-based algorithm to be used as a benchmark of true option values for testing the accuracy of the FFT and near-optimal FFT methods, especially for large-dimensional models. One of the key points is that the simulation is done primarily with respect to the underlying Markov chain. Note that (see Buffington and Elliott [6]), for a given realization of the underlying Markov chain, the model parameters (rate of return, volatility, and riskfree interest rate) can be determined. Consequently, the option price associated with the realization can be exactly calculated by the usual Black-Scholes formula in which the volatility and the interest rate are replaced by the sample path values. Therefore, it is only required to sample the Markov chain trajectories in order to carry out the Monte Carlo simulation. For this reason, we call the approach a semi-Monte Carlo simulation. A numerical example shows that the semi-Monte Carlo simulation outperforms the other approaches.

The rest of the paper is organized as follows. Section 2 begins with risk-neutral valuation for European option, where the asset price follows a regime-switching diffusion. The FFT approach is then developed. Section 3 presents a two-time-scale setup of the option pricing problem for the regime-switching model when the modulating Markov chain has a large state space. Also presented is near optimality for the FFT. As the small parameter diminishes, convergence of the option price is proved. In Section 4, we develop a novel semi-Monte Carlo simulation algorithm that can be used to yield benchmark values in numerical experiments. Numerical results are reported in Section 5. Finally, Section 6 provides further remarks and concludes the paper. 


\section{FFT approach for regime-switching option pricing}

In this section, we generalize the FFT technique introduced by Carr and Madan in [7] for option pricing to the regime-switching model and develop the FFT scheme.

2.1. Regime-switching model and risk-neutral option pricing. Suppose that $(\Omega, \mathscr{F}, \mathscr{P})$ is the underlying probability space, upon which all the stochastic processes below are defined. Let $\alpha(t)$ be a finite-state continuous time Markov chain with state space $M=$ $\{1, \ldots, m\}$, which may represent general market trends and/or other economic factors (also called "state of the world" or regime). For example, when $m=2, \alpha(t)=1$ denotes an uptrend and $\alpha(t)=2$ a downtrend.

Suppose that $S(t)$, the price of the underlying asset, satisfies a stochastic differential equation

$$
\frac{d S(t)}{S(t)}=v(\alpha(t)) d t+\sigma(\alpha(t)) d B(t), \quad t \geq 0,
$$

where $S(0)=S_{0}>0$ is the initial price, $B(t)$ is a one-dimensional real-valued standard Brownian motion independent of $\alpha(t)$, and $\nu(\alpha(t))$ and $\sigma(\alpha(t))$ are the rates of expected return and volatility of the asset, respectively. We assume that $\sigma(j)>0$, for each $j \in \mathcal{M}$. Assume that the instantaneous risk-free interest rate also depends on $\alpha(t)$ and use $r(\alpha(t))$ in the paper.

A standard methodology for option pricing is the risk-neutral valuation. The idea is to derive a suitable probability space upon which the discounted asset price process becomes a martingale; this probability space is usually called the risk neutral world and the associated probability measure is referred to as the risk-neutral or equivalent martingale measure. As a consequence, the fair value of an option is expressed as the expected value of the discounted option payoff with respect to this equivalent martingale measure.

Risk-neutral valuation is adopted for regime-switching models (see Yaol et al. [21], Guo [15]). Yaol et al. [21] provided a generalized Girsanov theorem for Markov modulated processes and derived an equivalent martingale measure $\widetilde{P}$ under which (2.1) becomes

$$
\frac{d S(t)}{S(t)}=\mu(\alpha(t)) d t+\sigma(\alpha(t)) d \widetilde{B}(t), \quad t \geq 0
$$

where $\widetilde{B}(t)$ is a standard $\widetilde{\mathscr{P}}$-Brownian motion independent of $\alpha(t), \mu(\alpha(t))$ denotes the risk-free drift rate. Note that we use $\mu(\alpha(t))$, not $r(\alpha(t))$, for the drift in (2.2). This is because the drift in the risk-neutral price process may not be equal to the risk-free interest rate. For example, a dividend rate (if nonzero) should be subtracted from the interest rate. Also as discussed by Guo [15], to have a complete market, Arrow-Debreu securities related to the switching cost need to be used. This results in an extra component that is subtracted from the interest rate. We refer the reader to Yaol et al. [21], Guo [15] for detailed discussions and technical proofs concerning the regime-switching risk-neutral valuation. 
From now on, we will work with the risk-neutral world $(\Omega, \mathscr{F}, \tilde{\mathscr{P}})$. Throughout the rest of the paper, all expectations are taken with respect to the risk-neutral measure $\tilde{\mathscr{P}}$. Let

$$
X(t)=\int_{0}^{t}\left(\mu(\alpha(s))-\frac{1}{2} \sigma^{2}(\alpha(s))\right) d s+\int_{0}^{t} \sigma(\alpha(s)) d \widetilde{B}(s), \quad t \geq 0 .
$$

Then the solution to (2.2) is given by

$$
S(t)=S_{0} \exp [X(t)]
$$

Consider an European call option written on $S(t)$ with strike $K>0$ and maturity $T>0$. By the risk-neutral valuation principle, the option price $C(K)$ is given by

$$
C(K)=E\left\{\exp \left(-\int_{0}^{T} r(\alpha(t)) d t\right)\left(S_{0} e^{X(T)}-K\right)^{+}\right\}
$$

Let $k=\ln \left(K / S_{0}\right)$. Then (2.2) can be written as

$$
C(k)=S_{0} E\left\{\exp \left(-\int_{0}^{T} r(\alpha(t)) d t\right)\left(e^{X(T)}-e^{k}\right)^{+}\right\}
$$

Note that we use $k$ (a modified log strike) instead of the log strike itself to ensure that $k=0$ will be always corresponding to the at-the-money case.

2.2. Fourier transform of the option price. Note that the call price $C(k)$ does not decay to 0 as $k$ goes to $-\infty$. Hence we cannot directly take its Fourier transform. Carr and Madan [7] introduced an extra exponential term to make the modified function be square integrable with respect to $k$ over $(-\infty, \infty)$, whose Fourier transform is well defined and also square integrable. We use this idea here for the regime-switching model.

Define a modified price function $c(k)$ by

$$
c(k)=e^{\rho k} \frac{C(k)}{S_{0}}, \quad-\infty<k<\infty,
$$

where $\rho>0$ is a prespecified positive number (damping factor). We derive an explicit formula for the Fourier transform of $c(k)$ next.

Let $\mathscr{F}_{T}$ be the $\sigma$-algebra generated by the Markov chain $\alpha(t), 0 \leq t \leq T$, that is, $\mathscr{F}_{T}=$ $\sigma\{\alpha(t), 0 \leq t \leq T\}$. Let $f_{\mathscr{F}_{T}}(x)$ be the conditional density function of $X(T)$ given $\mathscr{F}_{T}$. Define

$$
L_{T}=\int_{0}^{T} \mu(\alpha(t)) d t, \quad V_{T}=\int_{0}^{T} \sigma^{2}(\alpha(t)) d t, \quad R_{T}=\int_{0}^{T} r(\alpha(t)) d t .
$$


6 Option pricing in a regime-switching model using FFT

Then the Fourier transform of $c(k)$ is calculated as follows:

$$
\begin{aligned}
\psi(u) & =\int_{-\infty}^{\infty} e^{i u k} c(k) d k \\
& =\int_{-\infty}^{\infty} e^{i u k} e^{\rho k} E\left\{e^{-R_{T}}\left(e^{X(T)}-e^{k}\right)^{+}\right\} d k \\
& =E\left\{\int_{-\infty}^{\infty} e^{i u k} e^{\rho k} e^{-R_{T}} E\left\{\left(e^{X(T)}-e^{k}\right)^{+} \mid \mathscr{F}_{T}\right\} d k\right\} \\
& =E\left\{\int_{-\infty}^{\infty} e^{i u k} e^{\rho k} e^{-R_{T}} \int_{k}^{\infty}\left(e^{x}-e^{k}\right) f_{\mathscr{F}_{T}}(x) d x d k\right\} \\
& =E\left\{\int_{-\infty}^{\infty} e^{-R_{T}} f_{\mathscr{F}_{T}}(x) \int_{-\infty}^{x}\left(e^{x} e^{(\rho+i u) k}-e^{(1+\rho+i u) k}\right) d k d x\right\} \\
& =E\left\{\int_{-\infty}^{\infty} e^{-R_{T}} f_{\mathscr{F}_{T}}(x)\left(\frac{e^{(1+\rho+i u) x}}{\rho+i u}-\frac{e^{(1+\rho+i u) x}}{1+\rho+i u}\right) d x\right\} \\
& =E\left\{e^{-R_{T}}\left(\frac{\phi_{\mathscr{F}_{T}}(u-i(1+\rho))}{\rho+i u}-\frac{\phi_{\mathscr{F}_{T}}(u-i(1+\rho))}{1+\rho+i u}\right)\right\} \\
& =\frac{E\left\{e^{-R_{T}} \phi_{\mathscr{F}_{T}}(u-i(1+\rho))\right\}}{\rho^{2}+\rho-u^{2}+i(1+2 \rho) u},
\end{aligned}
$$

where

$$
\phi_{\mathscr{F}_{T}}(u)=E\left\{e^{i u X(T)} \mid \mathscr{F}_{T}\right\}=\int_{-\infty}^{\infty} e^{i u x} f_{\mathscr{F}_{T}}(x) d x
$$

is the conditional characteristic function of $X(T)$ given $\mathscr{F}_{T}$.

Note that given $\mathscr{F}_{T}, X(T)$ has Gaussian distribution with mean $\left(L_{T}-(1 / 2) V_{T}\right)$ and variance $V_{T}$. It follows that

$$
\phi_{\mathscr{F}_{T}}(u)=\exp \left(i u\left(L_{T}-\frac{1}{2} V_{T}\right)-\frac{1}{2} u^{2} V_{T}\right)
$$

Using (2.11) in (2.9), we have

$$
\begin{aligned}
\psi(u)=\frac{1}{\rho^{2}+\rho-u^{2}+i(1+2 \rho) u} E\{ & \exp \left((1+\rho)\left(L_{T}+\frac{1}{2} \rho V_{T}\right)-R_{T}\right. \\
& \left.\left.-\frac{1}{2} u^{2} V_{T}+i u\left(L_{T}+\left(\frac{1}{2}+\rho\right) V_{T}\right)\right)\right\} .
\end{aligned}
$$

To derive an explicit formula for $\psi(u)$, it is necessary to calculate the expectation with respect to the random variables $L_{T}, V_{T}$, and $R_{T}$. To this end, let

$$
T_{j}=\int_{0}^{T} I_{\{\alpha(t)=j\}} d t, \quad j \in \mathcal{M},
$$


be the sojourn time of the Markov chain $\alpha(t)$ in state $j$ during the interval $[0, T]$. Then $\sum_{j=1}^{m} T_{j}=T$. It follows that

$$
\begin{gathered}
L_{T}=\sum_{j=1}^{m-1}(\mu(j)-\mu(m)) T_{j}+\mu(m) T, \\
V_{T}=\sum_{j=1}^{m-1}\left(\sigma^{2}(j)-\sigma^{2}(m)\right) T_{j}+\sigma^{2}(m) T, \\
R_{T}=\sum_{j=1}^{m-1}(r(j)-r(m)) T_{j}+r(m) T .
\end{gathered}
$$

Using (2.14) in (2.12), we obtain that

$$
\psi(u)=\frac{1}{\rho^{2}+\rho-u^{2}+i(1+2 \rho) u} \exp (B(u) T) E\left\{\exp \left(i \sum_{j=1}^{m-1} A(u, j) T_{j}\right)\right\}
$$

where for $j=1, \ldots, m-1$,

$$
\begin{gathered}
A(u, j)=\left[(\mu(j)-\mu(m))+\left(\frac{1}{2}+\rho\right)\left(\sigma^{2}(j)-\sigma^{2}(m)\right)\right] u+\frac{1}{2} u^{2}\left(\sigma^{2}(j)-\sigma^{2}(m)\right) i \\
+\left[(r(j)-r(m))-(1+\rho)(\mu(j)-\mu(m))-\frac{1}{2} \rho(1+\rho)\left(\sigma^{2}(j)-\sigma^{2}(m)\right)\right] i, \\
B(u)=i u\left[\mu(m)+\left(\frac{1}{2}+\rho\right) \sigma^{2}(m)\right]-\frac{1}{2} u^{2} \sigma^{2}(m)+(1+\rho) \mu(m)-r(m)+\frac{1}{2} \rho(1+\rho) \sigma^{2}(m) .
\end{gathered}
$$

Therefore, the determination of $\psi(u)$ reduces to calculating the characteristic function of the random vector $\left(T_{1}, \ldots, T_{m-1}\right)^{\prime}$.

Two-state case. Consider $m=2$. Let the generator of the Markov chain $\alpha(\cdot)$ be given by

$$
Q=\left(\begin{array}{cc}
-\lambda_{1} & \lambda_{1} \\
\lambda_{2} & -\lambda_{2}
\end{array}\right), \quad \lambda_{1}, \lambda_{2}>0
$$

where $\lambda_{1}$ is the jump rate from state 1 to state 2 and $\lambda_{2}$ is the jump rate from state 2 to state 1 . In this case, we need to find the characteristic function of $T_{1}$, the sojourn time in state 1 .

Assume the initial state $\alpha(0)=j_{0}$. Define

$$
\phi_{j_{0}}(\theta, T)=E\left(e^{i \theta T_{1}} \mid \alpha(0)=j_{0}\right), \quad j_{0}=1,2
$$


Then $\phi_{1}(\theta, T)$ and $\phi_{2}(\theta, T)$ satisfy the following system of integral equations (see Guo [15]):

$$
\begin{gathered}
\phi_{1}(\theta, T)=e^{i \theta T} e^{-\lambda_{1} T}+\int_{0}^{T} e^{i \theta t} \phi_{2}(\theta, T-t) \lambda_{1} e^{-\lambda_{1} t} d t, \\
\phi_{2}(\theta, T)=e^{-\lambda_{2} T}+\int_{0}^{T} \phi_{1}(\theta, T-t) \lambda_{2} e^{-\lambda_{2} t} d t .
\end{gathered}
$$

Taking Laplace transform, we obtain the following system of algebraic equations:

$$
\begin{gathered}
\mathscr{L}\left\{\phi_{1}(\theta, T)\right\}=\frac{1}{s+\lambda_{1}-i \theta}+\frac{\lambda_{1}}{s+\lambda_{1}-i \theta} \mathscr{L}\left\{\phi_{2}(\theta, T)\right\}, \\
\mathscr{L}\left\{\phi_{2}(\theta, T)\right\}=\frac{1}{s+\lambda_{2}}+\frac{\lambda_{2}}{s+\lambda_{2}} \mathscr{L}\left\{\phi_{1}(\theta, T)\right\} .
\end{gathered}
$$

Solving the pair of equations yields

$$
\begin{aligned}
& \mathscr{L}\left\{\phi_{1}(\theta, T)\right\}=\frac{s+\lambda_{1}+\lambda_{2}}{s^{2}+\left(\lambda_{1}+\lambda_{2}-i \theta\right) s-i \theta \lambda_{2}}, \\
& \mathscr{L}\left\{\phi_{2}(\theta, T)\right\}=\frac{s+\lambda_{1}+\lambda_{2}-i \theta}{s^{2}+\left(\lambda_{1}+\lambda_{2}-i \theta\right) s-i \theta \lambda_{2}} .
\end{aligned}
$$

Taking inverse Laplace transform, we have

$$
\begin{gathered}
\phi_{1}(\theta, T)=\frac{1}{s_{1}-s_{2}}\left(\left(s_{1}+\lambda_{1}+\lambda_{2}\right) e^{s_{1} T}-\left(s_{2}+\lambda_{1}+\lambda_{2}\right) e^{s_{2} T}\right), \\
\phi_{2}(\theta, T)=\frac{1}{s_{1}-s_{2}}\left(\left(s_{1}+\lambda_{1}+\lambda_{2}-i \theta\right) e^{s_{1} T}-\left(s_{2}+\lambda_{1}+\lambda_{2}-i \theta\right) e^{s_{2} T}\right),
\end{gathered}
$$

where $s_{1}$ and $s_{2}$ are the two roots of the equation

$$
s^{2}+\left(\lambda_{1}+\lambda_{2}-i \theta\right) s-i \theta \lambda_{2}=0 .
$$

The Fourier transform (2.15) in this case is given by

$$
\psi(u)=\frac{\exp (B(u) T) \phi_{j_{0}}(A(u), T)}{\rho^{2}+\rho-u^{2}+i(1+2 \rho) u},
$$

where

$$
\begin{aligned}
A(u):= & A(u, 1) \\
= & {\left[(\mu(1)-\mu(2))+\left(\frac{1}{2}+\rho\right)\left(\sigma^{2}(1)-\sigma^{2}(2)\right)\right] u+\frac{1}{2} u^{2}\left(\sigma^{2}(1)-\sigma^{2}(2)\right) i } \\
& +\left[(r(1)-r(2))-(1+\rho)(\mu(1)-\mu(2))-\frac{1}{2} \rho(1+\rho)\left(\sigma^{2}(1)-\sigma^{2}(2)\right)\right] i, \\
B(u)= & i u\left[\mu(2)+\left(\frac{1}{2}+\rho\right) \sigma^{2}(2)\right]-\frac{1}{2} u^{2} \sigma^{2}(2)+(1+\rho) \mu(2)-r(2)+\frac{1}{2} \rho(1+\rho) \sigma^{2}(2) .
\end{aligned}
$$


R. H. Liu et al. 9

General case $(m \geq 2)$. Let the generator of the Markov chain $\alpha(\cdot)$ be given by an $m \times m$ matrix $Q=\left(q_{i j}\right)_{m \times m}$ such that $q_{i j} \geq 0$ for $i \neq j$ and $\sum_{j=1}^{m} q_{i j}=0$ for each $i \in \mathcal{M}$. Let

$$
\mathbf{I}(t)=\left(I_{\{\alpha(t)=1\}}, I_{\{\alpha(t)=2\}}, \ldots, I_{\{\alpha(t)=m\}}\right)^{\prime} \in \mathbb{R}^{m \times 1}
$$

denote the vector of indicator functions. Then it is shown by Yin and Zhang [23, Lemma 2.4, Chapter 2] that

$$
\mathbf{I}(t)-\int_{0}^{t} Q^{\prime} \mathbf{I}(s) d s
$$

is a martingale, where $Q^{\prime}$ denotes the transpose of $Q$.

To determine the characteristic function

$$
E\left\{\exp \left(i \sum_{j=1}^{m-1} \theta_{j} T_{j}\right)\right\}=E\left\{\exp \left(i \sum_{j=1}^{m-1} \theta_{j} \int_{0}^{T} I_{\{\alpha(t)=j\}} d t\right)\right\},
$$

define a random vector

$$
\mathbf{Z}(T)=\left(z_{1}(T), z_{2}(T), \ldots, z_{m}(T)\right)^{\prime} \in \mathbb{R}^{m \times 1},
$$

where

$$
z_{j}(T)=\exp \left(i \sum_{j=1}^{m-1} \theta_{j} \int_{0}^{T} I_{\{\alpha(t)=j\}} d t\right) I_{\{\alpha(T)=j\}}, \quad j=1,2, \ldots, m .
$$

Using the martingale property (2.27), it can be shown as by Buffington and Elliott [6] that $E[\mathbf{Z}(T)]$ satisfies the following vector differential equation:

$$
\frac{d E[\mathbf{Z}(T)]}{d T}=\left(Q^{\prime}+i \operatorname{diag}\left(\theta_{1}, \theta_{2}, \ldots, \theta_{m-1}, 0\right)\right) E[\mathbf{Z}(T)], \quad E[\mathbf{Z}(0)]=\mathbf{I}(0),
$$

where $\operatorname{diag}\left(\theta_{1}, \theta_{2}, \ldots, \theta_{m-1}, 0\right)$ is the diagonal matrix with diagonal entries $\theta_{1}, \theta_{2}, \ldots, \theta_{m-1}$, 0 . The solution to $(2.31)$ is given by

$$
E[\mathbf{Z}(T)]=\exp \left(Q^{\prime}+i \operatorname{diag}\left(\theta_{1}, \theta_{2}, \ldots, \theta_{m-1}, 0\right)\right) \mathbf{I}(0) .
$$

Consequently, the characteristic function can be determined by

$$
\begin{aligned}
E\left\{\exp \left(i \sum_{j=1}^{m-1} \theta_{j} T_{j}\right)\right\} & =\sum_{j=1}^{m} E\left[z_{j}(T)\right]=\mathbb{1}_{m}^{\prime} E[\mathbf{Z}(T)] \\
& =\mathbb{1}_{m}^{\prime} \exp \left(Q^{\prime}+i \operatorname{diag}\left(\theta_{1}, \theta_{2}, \ldots, \theta_{m-1}, 0\right)\right) \mathbf{I}(0),
\end{aligned}
$$

where $\mathbb{1}_{m}=(1, \ldots, 1)^{\prime} \in \mathbb{R}^{m \times 1}$.

Setting $\theta_{j}=A(u, j)$ in (2.33) and then using the result in (2.15), we obtain the Fourier transform $\psi(u)$, which can then be used in the inverse transform to determine the option price. 
2.3. FFT algorithm for option pricing. We adopt the approach introduced by Carr and Madan [7]. Recall that the discrete Fourier transform of a given sequence $\left\{f_{j}\right\}_{j=0}^{N-1}$ is defined by

$$
F_{l}=\sum_{j=0}^{N-1} e^{-i(2 \pi / N) l j} f_{j}, \quad l=0,1, \ldots, N-1 .
$$

It is typical to choose $N$ as a power of 2 when the FFT procedure is used to calculate the transform $\left\{F_{l}\right\}_{l=0}^{N-1}$.

Given the transform function $\psi(u)$, the modified option price $c(k)$ can be obtained by the inverse Fourier transform

$$
c(k)=\frac{1}{2 \pi} \int_{-\infty}^{\infty} e^{-i u k} \psi(u) d u=\frac{1}{\pi} \int_{0}^{\infty} e^{-i u k} \psi(u) d u,
$$

and the option price is, in view of (2.7), $C(k)=e^{-\rho k} S_{0} c(k)$.

Set $u_{j}=j \Delta_{u}, j=0,1, \ldots, N-1$, where $\Delta_{u}$ is the grid size in the variable $u$. Then (2.35) can be approximated by the following summation:

$$
c(k) \approx \frac{1}{\pi} \sum_{j=0}^{N-1} e^{-i u_{j} k} \psi\left(u_{j}\right) \Delta_{u}
$$

Next, let $\Delta_{k}$ be the grid size in $k$ and choose a grid along the modified log strike $k$ as below:

$$
k_{l}=\left(l-\frac{N}{2}\right) \Delta_{k}, \quad l=0,1, \ldots, N-1
$$

Then

$$
c\left(k_{l}\right) \approx \frac{1}{\pi} \sum_{j=0}^{N-1} e^{-i j l \Delta_{u} \Delta_{k}} e^{i j(N / 2) \Delta_{u} \Delta_{k}} \psi\left(j \Delta_{u}\right) \Delta_{u}, \quad l=0,1, \ldots, N-1 .
$$

If we set

$$
\Delta_{u} \Delta_{k}=\frac{2 \pi}{N}
$$

then we have

$$
c\left(k_{l}\right) \approx \frac{\Delta_{u}}{\pi} \sum_{j=0}^{N-1} e^{-i j l(2 \pi / N)} e^{i j \pi} \psi\left(j \Delta_{u}\right), \quad l=0,1, \ldots, N-1 .
$$

Using Simpson's rule for numerical integration, define a sequence of weighting factors by

$$
w(j)= \begin{cases}\frac{1}{3}, & \text { if } j=0 \\ \frac{4}{3}, & \text { if } j \text { is odd } \\ \frac{2}{3}, & \text { if } j \text { is even. }\end{cases}
$$


R. H. Liu et al. 11

Then

$$
c\left(k_{l}\right) \approx \frac{\Delta_{u}}{\pi} \sum_{j=0}^{N-1} e^{-i j l(2 \pi / N)} e^{i j \pi} \psi\left(j \Delta_{u}\right) w(j), \quad l=0,1, \ldots, N-1 .
$$

Comparing (2.42) with (2.34), it is easily seen that $\left\{c\left(k_{l}\right)\right\}_{l=0}^{N-1}$ can be obtained by taking the Fourier transform of the sequence $\left\{e^{i j \pi} \psi\left(j \Delta_{u}\right) w(j)\right\}_{j=0}^{N-1}$.

Remark 2.1. In order to apply the FFT procedure, a discrete sum (2.36) is used to approximate the continuous infinite integral (2.35). This will cause approximation errors in option values. For models without regime-switching, Lee [18] developed upper bounds for the truncation and discretization errors of the approximation. However, numerical results show that the actual errors in option prices frequently are much smaller than those bounds. Our numerical experiments in Section 5 (see Table 5.1) show that for the regimeswitching option valuation, using the chosen grid sizes, the effect of this discretization error is nearly unnoticeable.

\section{A near-optimal approach using a two-time-scale Markov chain}

In order to find the Fourier transform $\psi(u)$, it is necessary to solve the system of $m$ differential equations (2.31) (or equivalently, to calculate the matrix exponential (2.33)). When the number of states $m$ of the Markov chain is large, the computational complexity becomes a main concern. To overcome the difficulty, we develop an approximation approach using FFT and a two-time-scale Markov chain structure. We prove that this approach is near optimal in the sense that the approximation of the prices converges to the true prices as the time-scale parameter $\varepsilon$ approaches to zero.

3.1. A two-time-scale Markov chain. To reveal the explicit dependence on a small positive number $\varepsilon$, in this section, we use $\alpha^{\varepsilon}(\cdot)$ instead of $\alpha(\cdot)$ for the driving Markov chain. Let $Q^{\varepsilon}$ be its generator and assume $Q^{\varepsilon}$ has the following decomposition form:

$$
Q^{\varepsilon}=\frac{1}{\varepsilon} \widetilde{Q}+\hat{Q}=\frac{1}{\varepsilon}\left(\begin{array}{lll}
\widetilde{Q}^{1} & & \\
& \ddots & \\
& & \widetilde{Q}^{l}
\end{array}\right)+\widehat{Q},
$$

where, for each $k=1, \ldots, l, \widetilde{Q}^{k}$ is a generator with dimension $m_{k} \times m_{k}$, and $\hat{Q}$ is a generator with dimension $m \times m$. We have $m_{1}+\cdots+m_{l}=m$. Suppose that, for $k=1, \ldots, l, \widetilde{Q}^{k}$ are irreducible. Then we may rewrite the state space of $\alpha^{\varepsilon}(\cdot)$ as

$$
\mathcal{M}=M_{1} \cup \cdots \cup \mathcal{M}_{l}=\left\{s_{11}, \ldots, s_{1 m_{1}}\right\} \cup \cdots \cup\left\{s_{l 1}, \ldots, s_{l m_{l}}\right\},
$$

where for each $k=1, \ldots, l, M_{k}=\left\{s_{k 1}, \ldots, s_{k m_{k}}\right\}$ is the substate space corresponding to the $k$ th recurrent class. Let $\nu^{k}=\left(v_{1}^{k}, \ldots, v_{m_{k}}^{k}\right)$ denote the stationary distribution of $\widetilde{Q}^{k}$, that is, $\nu^{k}$ is the only solution to

$$
v^{k} \widetilde{Q}^{k}=0, \quad v^{k} \mathbb{1}_{m_{k}}=1,
$$


where $\mathbb{1}_{m_{k}}=(1, \ldots, 1)^{\prime} \in \mathbb{R}^{m_{k} \times 1}$. Define an aggregated process $\bar{\alpha}^{\varepsilon}(\cdot) \in\{1, \ldots, l\}$ by

$$
\bar{\alpha}^{\varepsilon}(t)=k, \quad \text { if } \alpha^{\varepsilon}(t) \in M_{k} .
$$

Next, define an $l \times l$ matrix $\bar{Q}$ by

$$
\bar{Q}=\operatorname{diag}\left(v^{1}, \ldots, v^{l}\right) \hat{Q} \operatorname{diag}\left(\mathbb{1}_{m_{1}}, \ldots, \mathbb{1}_{m_{l}}\right),
$$

where we use $\operatorname{diag}\left(A^{1}, \ldots, A^{l}\right)$ for the block diagonal matrix with entries $A^{1}, \ldots, A^{l}$. It was shown by Yin and Zhang [23, Theorems 7.2 and 7.4] that the following limit results hold.

(1) For any bounded and measurable deterministic functions $\beta_{k j}(t)$, the corresponding occupation times satisfy

$$
E\left(\int_{0}^{T}\left(I_{\left\{\alpha^{\varepsilon}(t)=s_{k j}\right\}}-v_{j}^{k} I_{\left\{\bar{\alpha}^{\varepsilon}(t)=k\right\}}\right) \beta_{k j}(t) d t\right)^{2}=O(\varepsilon) .
$$

(2) Given $T>0, \bar{\alpha}^{\varepsilon}(\cdot)$ converges weakly to $\bar{\alpha}(\cdot)$, a Markov chain with generator $\bar{Q}$ given in (3.5) and state space $\bar{M}=\{1, \ldots, l\}$.

3.2. Limit price model and near-optimal pricing method. Next, we define a limit price process using the limit Markov chain $\bar{\alpha}(\cdot)$. For $k=1, \ldots, l$, let

$$
\bar{\mu}(k)=\sum_{j=1}^{m_{k}} v_{j}^{k} \mu\left(s_{k j}\right), \quad \bar{\sigma}^{2}(k)=\sum_{j=1}^{m_{k}} v_{j}^{k} \sigma^{2}\left(s_{k j}\right), \quad \bar{r}(k)=\sum_{j=1}^{m_{k}} v_{j}^{k} r\left(s_{k j}\right) .
$$

Let $\bar{S}(t)$ be a diffusion process satisfying

$$
\frac{d \bar{S}(t)}{\bar{S}(t)}=\bar{\mu}(\bar{\alpha}(t)) d t+\bar{\sigma}(\bar{\alpha}(t)) d \widetilde{B}(t), \quad t \geq 0
$$

We assume that the Brownian motion $\widetilde{B}(t)$ is independent of the limit Markov chain $\bar{\alpha}(t)$, and the initial price $\bar{S}(0)=S_{0}$, the same initial value as in (2.2). Note that the limit price process $\bar{S}(t)$ is defined such that the parameters $\bar{\mu}(\cdot), \bar{\sigma}(\cdot)$, and $\bar{r}(\cdot)$ are replaced by their averages with respect to the corresponding stationary distributions. Noticeably, this process is still a switching diffusion process (i.e., a set of diffusions coupled by the limit Markov chain $\bar{\alpha}(\cdot))$. But the number of states is reduced from $m$ to $l$.

Consider an European call option written on the price process $\bar{S}(t)$ with the same maturity $T$ and strike $K$, whose value is given by

$$
\bar{C}(k)=E\left\{\exp \left(-\int_{0}^{T} \bar{r}(\bar{\alpha}(t)) d t\right)\left(S_{0} e^{\bar{X}(T)}-K\right)^{+}\right\}
$$

where

$$
\bar{X}(t)=\int_{0}^{t}\left(\bar{\mu}(\bar{\alpha}(s))-\frac{1}{2} \bar{\sigma}^{2}(\bar{\alpha}(s))\right) d s+\int_{0}^{t} \bar{\sigma}(\bar{\alpha}(s)) d \widetilde{B}(s), \quad t \geq 0 .
$$


Similar to (2.8), define

$$
\bar{L}_{T}=\int_{0}^{T} \bar{\mu}(\bar{\alpha}(t)) d t, \quad \bar{V}_{T}=\int_{0}^{T} \bar{\sigma}^{2}(\bar{\alpha}(t)) d t, \quad \bar{R}_{T}=\int_{0}^{T} \bar{r}(\bar{\alpha}(t)) d t .
$$

Let $\bar{c}(k)=e^{\rho k}\left(\bar{C}(k) / S_{0}\right)$. Then the Fourier transform of $\bar{c}(k)$ is given by

$$
\begin{aligned}
\bar{\psi}(u)=\frac{1}{\rho^{2}+\rho-u^{2}+i(1+2 \rho) u} E\{\exp ( & (1+\rho)\left(\bar{L}_{T}+\frac{1}{2} \rho \bar{V}_{T}\right)-\bar{R}_{T} \\
& \left.\left.-\frac{1}{2} u^{2} \bar{V}_{T}+i u\left(\bar{L}_{T}+\left(\frac{1}{2}+\rho\right) \bar{V}_{T}\right)\right)\right\},
\end{aligned}
$$

and $\bar{c}(k)$ is given by the inverse Fourier transform

$$
\bar{c}(k)=\frac{1}{\pi} \int_{0}^{\infty} e^{-i u k} \bar{\psi}(u) d u,
$$

which can be calculated by using the FFT presented in Section 2.

Remark 3.1. It should be pointed out that the purpose of defining the limit process (3.8) and the associated option price (3.9) is for developing a near-optimal method that can produce good approximation to the true (or original) option price with significantly reduced computation.

3.3. Convergence analysis. In this section, we show that as $\varepsilon \rightarrow 0$, the option price converges to the limit price. Note that corresponding to $\alpha^{\varepsilon}(\cdot)$, the functions $c(k), \psi(u)$, and so forth should all be indexed by $\varepsilon$ to highlight their $\varepsilon$ dependence. However, for notational simplicity, we suppress the $\varepsilon$-dependence in what follows. Thus, for example, by $c(k) \rightarrow \bar{c}(k)$, we mean that $c^{\varepsilon}(k) \rightarrow \bar{c}(k)$ as $\varepsilon \rightarrow 0$. To proceed, we introduce the following quantities associated with the aggregated process $\bar{\alpha}^{\varepsilon}(\cdot)$.

Let

$$
\bar{L}_{T}^{\varepsilon}=\int_{0}^{T} \bar{\mu}\left(\bar{\alpha}^{\varepsilon}(t)\right) d t, \quad \bar{V}_{T}^{\mathcal{\varepsilon}}=\int_{0}^{T} \bar{\sigma}^{2}\left(\bar{\alpha}^{\varepsilon}(t)\right) d t, \quad \bar{R}_{T}^{\varepsilon}=\int_{0}^{T} \bar{r}\left(\bar{\alpha}^{\varepsilon}(t)\right) d t .
$$

Define an auxiliary function $\bar{\psi}^{\varepsilon}(u)$ by

$$
\begin{aligned}
\bar{\psi}^{\varepsilon}(u)=\frac{1}{\rho^{2}+\rho-u^{2}+i(1+2 \rho) u} E\{\exp ( & (1+\rho)\left(\bar{L}_{T}^{\varepsilon}+\frac{1}{2} \rho \bar{V}_{T}^{\varepsilon}\right)-\bar{R}_{T}^{\varepsilon} \\
& \left.\left.-\frac{1}{2} u^{2} \bar{V}_{T}^{\varepsilon}+i u\left(\bar{L}_{T}^{\varepsilon}+\left(\frac{1}{2}+\rho\right) \bar{V}_{T}^{\varepsilon}\right)\right)\right\} .
\end{aligned}
$$

LEMMA 3.2. The following error bounds hold:

$$
\begin{aligned}
& E\left|L_{T}-\bar{L}_{T}^{\varepsilon}\right|=O(\sqrt{\varepsilon}), \\
& E\left|R_{T}-\bar{R}_{T}^{\varepsilon}\right|=O(\sqrt{\varepsilon}), \\
& E\left|V_{T}-\bar{V}_{T}^{\varepsilon}\right|=O(\sqrt{\varepsilon}) .
\end{aligned}
$$


14 Option pricing in a regime-switching model using FFT

Proof. In view of the definitions (2.8), (3.7), and (3.14), we have

$$
\begin{aligned}
\left|L_{T}-\bar{L}_{T}^{\varepsilon}\right| & =\left|\int_{0}^{T} \mu\left(\alpha^{\varepsilon}(t)\right) d u-\int_{0}^{T} \bar{\mu}\left(\bar{\alpha}^{\varepsilon}(t)\right) d t\right| \\
& =\left|\sum_{k=1}^{l} \sum_{j=1}^{m_{k}} \mu\left(s_{k j}\right) \int_{0}^{T} I_{\left\{\alpha^{\varepsilon}(t)=s_{k j}\right\}} d t-\sum_{k=1}^{l} \bar{\mu}(k) \int_{0}^{T} I_{\left\{\bar{\alpha}^{\varepsilon}(t)=k\right\}} d t\right| \\
& \leq \sum_{k=1}^{l}\left|\sum_{j=1}^{m_{k}} \mu\left(s_{k j}\right) \int_{0}^{T} I_{\left\{\alpha^{\varepsilon}(t)=s_{k j}\right\}} d t-\sum_{j=1}^{m_{k}} v_{j}^{k} \mu\left(s_{k j}\right) \int_{0}^{T} I_{\left\{\bar{\alpha}^{\varepsilon}(t)=k\right\}} d t\right| \\
& \leq \sum_{k=1}^{l} \sum_{j=1}^{m_{k}}\left|\int_{0}^{T}\left(I_{\left\{\alpha^{\varepsilon}(t)=s_{k j}\right\}}-v_{j}^{k} I_{\left\{\bar{\alpha}^{\varepsilon}(t)=k\right\}}\right) \mu\left(s_{k j}\right) d t\right| .
\end{aligned}
$$

In terms of the limit result (3.6), an application of the Cauchy-Schwarz inequality yields

$$
E\left|L_{T}-\bar{L}_{T}^{\varepsilon}\right| \leq \sum_{k=1}^{l} \sum_{j=1}^{m_{k}} E\left|\int_{0}^{T}\left(I_{\left\{\alpha^{\varepsilon}(t)=s_{k j}\right\}}-v_{j}^{k} I_{\left\{\bar{\alpha}^{\varepsilon}(t)=k\right\}}\right) \mu\left(s_{k j}\right) d t\right|=O(\sqrt{\varepsilon}) .
$$

This proves (3.16). The other two inequalities can be obtained similarly.

Lemma 3.3. There is a positive number $\beta>0$ such that for $0 \leq u<\infty$,

$$
\left|\psi(u)-\bar{\psi}^{\varepsilon}(u)\right|=e^{-\beta u^{2}}\left(\frac{u^{2}+u+1}{u^{2}+\rho(1+\rho)}\right) O(\sqrt{\varepsilon}) .
$$

Proof. Let

$$
\begin{array}{cl}
X(u)=(1+\rho)\left(L_{T}+\frac{1}{2} \rho V_{T}\right)-R_{T}-\frac{1}{2} V_{T} u^{2}, & Y(u)=u\left(L_{T}+\left(\frac{1}{2}+\rho\right) V_{T}\right), \\
\bar{X}^{\varepsilon}(u)=(1+\rho)\left(\bar{L}_{T}^{\varepsilon}+\frac{1}{2} \rho \bar{V}_{T}^{\varepsilon}\right)-\bar{R}_{T}^{\varepsilon}-\frac{1}{2} \bar{V}_{T}^{\varepsilon} u^{2}, & \bar{Y}^{\varepsilon}(u)=u\left(\bar{L}_{T}^{\varepsilon}+\left(\frac{1}{2}+\rho\right) \bar{V}_{T}^{\varepsilon}\right) .
\end{array}
$$

Let

$$
\begin{array}{rlrl}
\mu_{\max } & =\max _{i \in \mathcal{M}}\{\mu(i)\}, & \bar{\mu}_{\max }=\max _{i \in \overline{\mathcal{M}}}\{\bar{\mu}(i)\}, \\
\sigma_{\max }^{2}=\max _{i \in \mathcal{M}}\left\{\sigma^{2}(i)\right\}, & \bar{\sigma}_{\max }^{2}=\max _{i \in \overline{\mathcal{M}}}\left\{\bar{\sigma}^{2}(i)\right\}, \\
\sigma_{\min }^{2}=\min _{i \in \mathcal{M}}\left\{\sigma^{2}(i)\right\}, & \bar{\sigma}_{\min }^{2}=\min _{i \in \overline{\mathcal{M}}}\left\{\bar{\sigma}^{2}(i)\right\} .
\end{array}
$$

Then we have

$$
\bar{\mu}_{\max } \leq \mu_{\max }, \quad \bar{\sigma}_{\max }^{2} \leq \sigma_{\max }^{2}, \quad \bar{\sigma}_{\min }^{2} \geq \sigma_{\min }^{2} .
$$

Then

$$
\begin{gathered}
L_{T} \leq \mu_{\max } T, \quad \bar{L}_{T}^{\varepsilon} \leq \bar{\mu}_{\max } T \leq \mu_{\max } T, \\
\sigma_{\min }^{2} T \leq V_{T} \leq \sigma_{\max }^{2} T, \quad \sigma_{\min }^{2} T \leq \bar{\sigma}_{\min }^{2} T \leq \bar{V}_{T}^{\varepsilon} \leq \bar{\sigma}_{\max }^{2} T \leq \sigma_{\max }^{2} T .
\end{gathered}
$$


It follows that

$$
\begin{gathered}
X(u) \leq(1+\rho)\left(\mu_{\max }+\frac{1}{2} \rho \sigma_{\max }^{2}\right) T-\frac{1}{2} \sigma_{\min }^{2} u^{2}, \\
\bar{X}^{\varepsilon}(u) \leq(1+\rho)\left(\mu_{\max }+\frac{1}{2} \rho \sigma_{\max }^{2}\right) T-\frac{1}{2} \sigma_{\min }^{2} u^{2},
\end{gathered}
$$

for $u \geq 0$.

In view of (2.12) and (3.15), we have

$$
\begin{aligned}
\left|\psi(u)-\bar{\psi}^{\varepsilon}(u)\right| & \leq \frac{E\left|\exp (X(u)+i Y(u))-\exp \left(\bar{X}^{\varepsilon}(u)+i \bar{Y}^{\varepsilon}(u)\right)\right|}{\left|\rho^{2}+\rho-u^{2}+i(1+2 \rho) u\right|} \\
& \leq \frac{E\left|\exp (X(u)+i Y(u))-\exp \left(\bar{X}^{\varepsilon}(u)+i \bar{Y}^{\varepsilon}(u)\right)\right|}{u^{2}+\rho(1+\rho)} .
\end{aligned}
$$

It is elementary to show that for any two complex numbers $x_{1}+i y_{1}$ and $x_{2}+i y_{2}$,

$$
\left|\exp \left(x_{1}+i y_{1}\right)-\exp \left(x_{2}+i y_{2}\right)\right| \leq \sqrt{2} \max \left\{e^{x_{1}}, e^{x_{2}}\right\}\left(\left|x_{1}-x_{2}\right|+\left|y_{1}-y_{2}\right|\right) .
$$

Therefore,

$$
\left|\psi(u)-\bar{\psi}^{\varepsilon}(u)\right| \leq C e^{-\beta u^{2}} \frac{E\left|X(u)-\bar{X}^{\varepsilon}(u)\right|+E\left|Y(u)-\bar{Y}^{\varepsilon}(u)\right|}{u^{2}+\rho(1+\rho)},
$$

where

$$
\beta:=\frac{1}{2} \sigma_{\min }^{2}>0, \quad C:=\sqrt{2} \exp \left((1+\rho)\left(\mu_{\max }+\frac{1}{2} \rho \sigma_{\max }^{2}\right) T\right) .
$$

Using Lemma 3.2, we have

$$
\begin{aligned}
E\left|X(u)-\bar{X}^{\varepsilon}(u)\right| \leq & (1+\rho) E\left|L_{T}-\bar{L}_{T}^{\varepsilon}\right|+\frac{1}{2} \rho(1+\rho) E\left|V_{T}-\bar{V}_{T}^{\varepsilon}\right| \\
& +E\left|R_{T}-\bar{R}_{T}^{\varepsilon}\right|+\frac{1}{2} u^{2} E\left|V_{T}-\bar{V}_{T}^{\varepsilon}\right| \\
\leq & (1+\rho) O(\sqrt{\varepsilon})+\frac{1}{2} \rho(1+\rho) O(\sqrt{\varepsilon})+O(\sqrt{\varepsilon})+\frac{1}{2} u^{2} O(\sqrt{\varepsilon}) \\
= & \left(1+u^{2}\right) O(\sqrt{\varepsilon}) .
\end{aligned}
$$

Similarly,

$$
E\left|Y(u)-\bar{Y}^{\varepsilon}(u)\right|=u O(\sqrt{\varepsilon}) .
$$

Combining (3.28), (3.30), and (3.31) yields (3.20).

Theorem 3.4. As $\varepsilon \rightarrow 0$, the Fourier transform $\psi(u)$ converges to the limit Fourier transform $\bar{\psi}(u)$, that is,

$$
\lim _{\varepsilon \rightarrow 0} \psi(u)=\bar{\psi}(u)
$$


Proof. Using the auxiliary function $\bar{\psi}^{\varepsilon}(u)$, we have

$$
|\psi(u)-\bar{\psi}(u)| \leq\left|\psi(u)-\bar{\psi}^{\varepsilon}(u)\right|+\left|\bar{\psi}^{\varepsilon}(u)-\bar{\psi}(u)\right| \text {. }
$$

The first term in (3.33) goes to zero by Lemma 3.3. To show that the second term also goes to zero, we rewrite the random variables in $\bar{\psi}^{\varepsilon}(u)$ and $\bar{\psi}(u)$ in terms of the aggregated process $\bar{\alpha}^{\varepsilon}(\cdot)$ and the limit Markov chain $\bar{\alpha}(\cdot)$, respectively. Then it follows that

$$
\left|\bar{\psi}^{\varepsilon}(u)-\bar{\psi}(u)\right| \leq \frac{\left|E\left\{\exp \left(h\left(\bar{\alpha}^{\varepsilon}(\cdot)\right)\right)\right\}-E\{\exp (h(\bar{\alpha}(\cdot)))\}\right|}{u^{2}+\rho(1+\rho)},
$$

where

$$
\begin{aligned}
h\left(\bar{\alpha}^{\varepsilon}(\cdot)\right)=\sum_{k=1}^{l}[ & (1+\rho)\left(\bar{\mu}(k)+\frac{1}{2} \rho \bar{\sigma}^{2}(k)\right)-\bar{r}(k)-\frac{1}{2} \bar{\sigma}^{2}(k) u^{2} \\
& \left.+i u\left(\bar{\mu}(k)+\left(\frac{1}{2}+\rho\right) \bar{\sigma}^{2}(k)\right)\right] \int_{0}^{T} I_{\left\{\bar{\alpha}^{\varepsilon}(t)=k\right\}} d t,
\end{aligned}
$$

and $h(\bar{\alpha}(\cdot))$ is given by replacing $\bar{\alpha}^{\varepsilon}(t)$ with $\bar{\alpha}(t)$ in the above expression.

Using the weak convergence of $\bar{\alpha}^{\varepsilon}(\cdot)$ to $\bar{\alpha}(\cdot)$ (see [23, Theorem 7.4]), by virtue of the Skorohod representation (see, e.g., [24, Theorem 14.5]), and the dominated convergence theorem, we can show that

$$
E\left\{\exp \left(h\left(\bar{\alpha}^{\varepsilon}(\cdot)\right)\right)\right\} \longrightarrow E\{\exp (h(\bar{\alpha}(\cdot)))\},
$$

which, in turn, implies

$$
\bar{\psi}^{\varepsilon}(u) \longrightarrow \bar{\psi}(u)
$$

This completes the proof.

Corollary 3.5.

$$
\lim _{\varepsilon \rightarrow 0} c(k)=\bar{c}(k)
$$

Proof. As in the proof of Lemma 3.3, it is easy to show that

$$
|\psi(u)| \leq \frac{\exp \left((1+\rho)\left(\mu_{\max }+(1 / 2) \rho \sigma_{\max }^{2}\right) T-(1 / 2) \sigma_{\min }^{2} u^{2}\right)}{u^{2}+\rho(1+\rho)} .
$$

The right-hand side function is integrable. Applying the dominated convergence theorem, we have (3.38).

\section{A semi-Monte Carlo algorithm}

Monte Carlo simulations are frequently used when closed-form solutions are not available for complex stochastic problems. A Monte Carlo algorithm frequently serves as a benchmark for the "true value" used for testing other numerical methods. The benchmark value is obtained by running a great number of sample paths in simulating the 
Let $N$ be the number of replications.

For $n=1, \ldots, N$,

(1) obtain the $n$th sample path of $\alpha(t), 0 \leq t \leq T$,

(2) calculate $L_{T}, V_{T}$, and $R_{T}$ associated with the sample path,

(3) calculate the Black-Scholes price $C_{n}(K)$ for the $n$th sample path,

(4) calculate the average $C(K)=(1 / N) \sum_{n=1}^{N} C_{n}(K)$.

Algorithm 4.1

underlying stochastic dynamics. It is very time consuming and therefore not feasible for most practical use in real time.

Guo [15] used the CRR framework (Cox et al. [11]) to discretize the regime-switching model (2.2) and developed a binomial tree approach for option pricing. Fuh and Wang [14] presented a Monte Carlo simulation algorithm based on the discretized price model. As noted by Buffington and Elliott [6], for a given realization of the Markov chain $\alpha(\cdot)=$ $\{\alpha(t): 0 \leq t \leq T\}$, the corresponding option price can be calculated by the usual BlackScholes formula in which the volatility and the interest rate are replaced by the sample path values. In this section, we propose a semi-Monte Carlo algorithm for option pricing. This approach only takes random sampling of the Markov chain and then takes advantage of the availability of analytical formula (therefore exact) of the conditional price. The numerical results presented in this section demonstrate that it is superior than both the tree and the Monte Carlo simulation methods mentioned above.

Recall that from Section 2, $\mathscr{F}_{T}$ denotes the $\sigma$-algebra generated by the Markov chain $\alpha(t), 0 \leq t \leq T$. Then the call option price can be calculated by

$$
\begin{aligned}
C(K) & =E\left\{\exp \left(-\int_{0}^{T} r(\alpha(t)) d t\right)(S(T)-K)^{+}\right\} \\
& =E\left\{E\left[e^{-R_{T}}(S(T)-K)^{+} \mid \mathscr{F}_{T}\right]\right\} .
\end{aligned}
$$

The conditional expectation is given by the Black-Scholes formula, that is,

$$
E\left[e^{-R_{T}}(S(T)-K)^{+} \mid \mathscr{F}_{T}\right]=S_{0} e^{-\left(R_{T}-L_{T}\right)} N\left(d_{1}\left(L_{T}, V_{T}\right)\right)-K e^{-R_{T}} N\left(d_{2}\left(L_{T}, V_{T}\right)\right),
$$

where

$$
d_{1}\left(L_{T}, V_{T}\right)=\frac{\ln \left(S_{0} / K\right)+L_{T}+(1 / 2) V_{T}}{\sqrt{V_{T}}}, \quad d_{2}\left(L_{T}, V_{T}\right)=d_{1}\left(L_{T}, V_{T}\right)-\sqrt{V_{T}},
$$

and $N(\cdot)$ is the cumulative standard normal distribution function. We present Algorithm 4.1 for the semi-Monte Carlo simulation.

To implement the semi-Monte Carlo algorithm, we follow the method for sampling Markov chains by Yin and Zhang [23, Section 4, Chapter 2].

We consider a two-state $(m=2)$ example. When the underlying Markov chain $\alpha(\cdot)$ has only two states, an analytical formula in terms of an integral with respect to the Bessel function is developed by Guo [15] for the European call option prices. Fuh and Wang [14] considered a specific example and compared various methods (binomial tree, Monte 
TABLE 4.1. Comparison of semi-Monte Carlo with other methods.

\begin{tabular}{c|ccccc}
\hline$T$ (year) & Analytical & MC (error) & \multicolumn{1}{c}{ Tree (error) } & FW-method (error) & Semi-MC (error) \\
\hline 0.1 & 10.993 & $10.986(0.007)$ & $10.910(0.083)$ & $10.993(0.000)$ & $10.993(0.000)$ \\
0.2 & 12.164 & $12.151(0.013)$ & $12.257(-0.093)$ & $12.166(-0.002)$ & $12.165(-0.001)$ \\
0.5 & 15.614 & $15.592(0.022)$ & $15.497(0.117)$ & $15.639(-0.025)$ & $15.614(0.000)$ \\
1.0 & 20.721 & $20.681(0.040)$ & $20.740(-0.019)$ & $20.811(-0.091)$ & $20.721(0.000)$ \\
2.0 & 29.287 & $29.256(0.031)$ & $29.166(0.121)$ & $29.478(-0.191)$ & $29.291(-0.004)$ \\
3.0 & 36.476 & $36.426(0.050)$ & $36.219(0.257)$ & $36.689(-0.213)$ & $36.481(-0.005)$ \\
\hline
\end{tabular}

Carlo, and an approximation approach presented in their paper) with the analytical results. Here we consider the same example to compare the semi-Monte Carlo algorithm with those methods.

Example 4.1. The parameters in this example are $S_{0}=100, K=90, \lambda_{1}=\lambda_{2}=1.0, \mu(1)=$ $\mu(2)=r(1)=r(2)=0.1, \sigma(1)=0.2, \sigma(2)=0.3$. The initial state $\alpha(0)=1 . N=100000$ replications are used in the semi-Monte Carlo simulations. Table 4.1 lists the numerical results for a range of option expiry times.

In Table 4.1, the second column is the analytical prices, the third and fourth columns list the results obtained by the Monte Carlo and the binomial tree methods based on the discretized asset model, respectively, the fifth column has numbers obtained by the approximation approach presented by Fuh and Wang [14]. The results in those columns are obtained in [14]. The last column reports the numerical results by using the semi-Monte Carlo simulation algorithm presented in this section. The numbers in parentheses are the differences between the analytical prices (exact values) and the approximate numbers.

It is clear from Table 4.1 that the semi-Monte Carlo simulation outperforms the other three approximation methods. While most of the errors in the third to fifth columns exceed 2 cents (the worst case is about a quarter), all the errors in the last column are at most half cent, a clear indication of high accuracy. We also mention that Fuh and Wang [14] used 50000000 replications in their Monte Carlo simulations to obtain the numbers. We only used 100000 replications (1/50 of theirs) in the semi-Monte Carlo simulations but achieved a much higher degree of accuracy.

\section{Numerical experiments using FFT and near-optimal FFT}

In this section, we report numerical results of using FFT and near-optimal FFT for option pricing developed in this paper. We use the semi-Monte Carlo simulation results as benchmarks for comparisons. Two numerical examples are provided. The first one is concerned with the FFT method and the second one is for the near-optimal FFT.

In implementing the FFT, we choose the number of grid points $N=4096\left(2^{12}\right)$. That is, we invoke the FFT procedure to calculate 4096 option prices simultaneously. The grid size along the log strike price $k$ is set to be $\Delta_{k}=0.01$. Consequently, $\Delta_{u}=0.1534$ by (2.39). We choose the damping factor $\rho$ to be $\rho=1.0$ and use 100000 replications for the semiMonte Carlo simulations. All options considered in the examples have maturity $T=1$ (year). The initial asset price $S_{0}=\$ 100$. 
TABle 5.1. Option prices using FFT and semi-Monte Carlo simulations.

\begin{tabular}{c|cccc}
\hline $\ln \left(K / S_{0}\right)(K)$ & $\begin{array}{c}\text { Semi-MC } \\
\alpha(0)=1\end{array}$ & $\begin{array}{c}\text { FFT } \\
\alpha(0)=1\end{array}$ & $\begin{array}{c}\text { Semi-MC } \\
\alpha(0)=2\end{array}$ & $\begin{array}{c}\text { FFT } \\
\alpha(0)=2\end{array}$ \\
\hline$-0.3(74.082)$ & 34.774 & $34.774(0.000)$ & 34.742 & $34.742(0.000)$ \\
$-0.2(81.873)$ & 29.696 & $29.696(0.000)$ & 29.642 & $29.642(0.000)$ \\
$-0.1(90.484)$ & 24.764 & $24.764(0.000)$ & 24.688 & $24.689(-0.001)$ \\
$0(100)$ & 20.117 & $20.116(0.001)$ & 20.022 & $20.022(0.000)$ \\
$0.1(110.517)$ & 15.881 & $15.881(0.000)$ & 15.773 & $15.774(-0.001)$ \\
$0.2(122.140)$ & 12.158 & $12.157(0.001)$ & 12.042 & $12.043(-0.001)$ \\
$0.3(134.986)$ & 9.006 & $9.006(0.000)$ & 8.892 & $8.893(-0.001)$ \\
\hline
\end{tabular}

5.1. FFT example. We consider a two-state Markov chain model. The parameters are given by $\lambda_{1}=20, \lambda_{2}=30, \mu(1)=r(1)=0.05, \mu(2)=r(2)=0.1, \sigma(1)=0.5, \sigma(2)=0.3$. Note that unlike Example 4.1, in this model, the parameters $\mu, \sigma$, and $r$ all vary with different states. Large jump rates $\lambda_{1}$ and $\lambda_{2}$ are chosen so that the system switches frequently during the life of the options.

Table 5.1 reports the results for 7 call options with different strike prices (from deepin-the-money to at-the-money and to deep-out-of-money) obtained using FFT and the semi-Monte Carlo simulations. Column one lists the log strike (the strike) for the options. Columns two to five list the FFT and semi-Monte Carlo simulation prices for both $\alpha(0)=1$ and $\alpha(0)=2$. The numbers in parentheses are the differences between the two approaches. We can see that the differences are very small (1/10 cent).

We used a notebook PC with Celeron CPU $2.40 \mathrm{GHz}$ for the experiments. In each case $(\alpha(0)=1$ and $\alpha(0)=2)$, a single run of FFT algorithm produces 4096 option prices (each one with a different strike price and all other parameters are the same). We used the semiMonte Carlo simulations to calculate the prices of the same 4096 option prices. We found that it took about an hour for the semi-Monte Carlo simulation to finish the calculation, while it took only a few seconds to run the FFT algorithm. This shows the clear advantage of the FFT.

5.2. Near-optimal FFT example. We consider a four-state Markov chain $\alpha^{\varepsilon}(\cdot)$ with state space $\mathcal{M}=\left\{s_{11}, s_{12}, s_{21}, s_{22}\right\}$ and generator

$$
Q^{\varepsilon}=\frac{1}{\varepsilon}\left(\begin{array}{cccc}
-\lambda_{1} & \lambda_{1} & 0 & 0 \\
\lambda_{2} & -\lambda_{2} & 0 & 0 \\
0 & 0 & -\lambda_{1} & \lambda_{1} \\
0 & 0 & \lambda_{2} & -\lambda_{2}
\end{array}\right)+\left(\begin{array}{cccc}
-\mu_{1} & 0 & \mu_{1} & 0 \\
0 & -\mu_{1} & 0 & \mu_{1} \\
\mu_{2} & 0 & -\mu_{2} & 0 \\
0 & \mu_{2} & 0 & -\mu_{2}
\end{array}\right) \text {, }
$$

for some constants $\lambda_{1}, \lambda_{2}, \mu_{1}$, and $\mu_{2}$. The generator has two blocks. The stationary distributions are given by

$$
v^{1}=v^{2}=\left(\frac{\lambda_{2}}{\lambda_{1}+\lambda_{2}}, \frac{\lambda_{1}}{\lambda_{1}+\lambda_{2}}\right) .
$$


TABLE 5.2. Comparison between near-optimal FFT prices and benchmark prices $(\varepsilon=0.01)$.

\begin{tabular}{c|cccccc}
\hline \multirow{2}{*}{$\ln \left(K / S_{0}\right)(K)$} & $\begin{array}{c}\text { Limit- } \\
\text { FFT }\end{array}$ & $\begin{array}{c}\text { Semi- } \\
\text { MC }\end{array}$ & $\begin{array}{c}\text { Semi- } \\
\text { MC }\end{array}$ & $\begin{array}{c}\text { Limit- } \\
\text { FFT }\end{array}$ & $\begin{array}{c}\text { Semi- } \\
\text { MC }\end{array}$ & $\begin{array}{c}\text { Semi- } \\
\text { MC }\end{array}$ \\
& $\bar{\alpha}(0)=1$ & $\alpha(0)=s_{11}$ & $\alpha(0)=s_{12}$ & $\bar{\alpha}(0)=2$ & $\alpha(0)=s_{21}$ & $\alpha(0)=s_{22}$ \\
\hline$-0.3(74.082)$ & 36.935 & $36.942(0.007)$ & $36.929(-0.006)$ & 36.733 & $36.737(0.004)$ & $36.730(-0.003)$ \\
$-0.2(81.873)$ & 32.057 & $32.063(0.006)$ & $32.051(-0.006)$ & 31.734 & $31.738(0.004)$ & $31.728(-0.006)$ \\
$-0.1(90.484)$ & 27.275 & $27.282(0.007)$ & $27.268(-0.007)$ & 26.832 & $26.836(0.004)$ & $26.830(-0.002)$ \\
$0(100)$ & 22.709 & $22.716(0.007)$ & $22.700(-0.009)$ & 22.156 & $22.163(0.007)$ & $22.153(-0.003)$ \\
$0.1(110.517)$ & 18.469 & $18.476(0.007)$ & $18.459(-0.010)$ & 17.831 & $17.838(0.007)$ & $17.826(-0.005)$ \\
$0.2(122.140)$ & 14.648 & $16.655(0.007)$ & $14.637(-0.011)$ & 13.959 & $13.965(0.006)$ & $13.954(-0.005)$ \\
$0.3(134.986)$ & 11.312 & $11.320(0.008)$ & $11.303(-0.009)$ & 10.613 & $10.619(0.006)$ & $10.608(-0.005)$ \\
\hline
\end{tabular}

The generator of $\bar{\alpha}(\cdot)$, the limit Markov chain, is given by

$$
\bar{Q}=\left(\begin{array}{cc}
-\mu_{1} & \mu_{1} \\
\mu_{2} & -\mu_{2}
\end{array}\right) .
$$

The expected rate of return $\mu(\alpha)$, the volatility $\sigma(\alpha)$, and the risk-free interest rate $r(\alpha)$, at different states $\alpha=s_{k j} \in \mathcal{M}$, are chosen as

$$
\begin{array}{ccll}
\mu\left(s_{11}\right)=0.03, & \mu\left(s_{12}\right)=0.10, & \mu\left(s_{21}\right)=0.05, & \mu\left(s_{22}\right)=0.15, \\
\sigma\left(s_{11}\right)=80 \%, & \sigma\left(s_{12}\right)=30 \%, & \sigma\left(s_{21}\right)=60 \%, & \sigma\left(s_{22}\right)=20 \%, \\
r\left(s_{11}\right)=0.03, & r\left(s_{12}\right)=0.10, & r\left(s_{21}\right)=0.05, & r\left(s_{22}\right)=0.15 .
\end{array}
$$

The parameters in the generator $Q^{\varepsilon}$ are set to be

$$
\lambda_{1}=5.0, \quad \lambda_{2}=3.0, \quad \mu_{1}=4.0, \quad \mu_{2}=2.5, \quad \varepsilon=0.01 .
$$

We used the FFT to calculate the two-state limit prices as approximations to the true prices, and used semi-Monte Carlo simulations for the original four-state model to obtain benchmark prices. Table 5.2 reports the results on the 7 call options.

In Table 5.2, all but one option have errors within 1 penny. This clearly demonstrates the effectiveness of the near-optimal FFT approach. In some applications, projection for instance, where the exact numbers are not so critical but hundred and thousand options prices need to be estimated quickly, fast algorithms are especially needed. The nearoptimal FFT we presented in this paper is efficient and suitable for the intended applications since it can produce the results in a fast way with a very limited sacrifice in accuracy.

\section{Concluding remarks}

Fast Fourier transform (FFT) has been used for calculating option prices for a wide range of asset price models. In this paper, we extended the technique to the class of regimeswitching diffusion models and developed the FFT scheme. When the number of states of the driving Markov chain in the model is very large, the calculation of the characteristic 
function involved in the FFT approach becomes computationally intensive. To reduce the computational burden, we presented a near-optimal FFT approach based on a two-timescale structure of the underlying model. It was shown by both mathematical analysis and numerical experiments that the near-optimal values can be used to approximate the true option values with satisfactory accuracy. The salient feature of this approach is that it combines the speedup of FFT with the ability of structurally reducing the complexity of the two-time-scale modelling and that results in an effective and efficient numerical approximation algorithm for large-dimensional problems, which is a promising direction for future research.

\section{References}

[1] D. H. Bailey and P. N. Swarztrauber, The fractional Fourier transform and applications, SIAM Review 33 (1991), no. 3, 389-404.

[2] G. Barone-Adesi and R. Whaley, Efficient analytic approximation of American option values, Journal of Finance 42 (1987), 301-320.

[3] E. Benhamou, Fast Fourier transform for discrete Asian options, Journal of Computational Finance 6 (2002), no. 1.

[4] N. P. B. Bollen, Valuing options in regime-switching models, Journal of Derivatives 6 (1998), 38 49.

[5] E. O. Brigham, The Fast Fourier Transform and Applications, Prentice-Hall, New York, 1988.

[6] J. Buffington and R. J. Elliott, American options with regime switching, International Journal of Theoretical and Applied Finance 5 (2002), no. 5, 497-514.

[7] P. Carr and D. B. Madan, Option valuation using the fast Fourier transform, Journal of Computational Finance 2 (1998), no. 4, 61-73.

[8] P. Carr and L. Wu, Time changed Lévy processes and option pricing, Journal of Financial Economics 71 (2004), no. 1, 113-141.

[9] A. Černý, Introduction to fast Fourier transform in finance, Journal of Derivatives 12 (2004), no. 1, 73-88.

[10] K. Chourdakis, Option pricing using the fractional FFT, Journal of Computational Finance 8 (2004), no. 2, 1-18.

[11] J. Cox, S. Ross, and M. Rubinstein, Option pricing, a simplified approach, Journal of Financial Economics 7 (1979), 229-263.

[12] M. A. H. Dempster and S. S. G. Hong, Spread option valuation and the fast Fourier transform, Mathematical Finance-Bachelier Congress, 2000 (Paris) (H. German, D. Madan, S. R. Pliska, and T. Vorst, eds.), Springer Finance, Springer, Berlin, 2002, pp. 203-220.

[13] D. Duffie, D. Filipović, and W. Schachermayer, Affine processes and applications in finance, The Annals of Applied Probability 13 (2003), no. 3, 984-1053.

[14] C.-D. Fuh and R.-H. Wang, Option pricing in a Black-Scholes model with Markov switching, Working Paper, Institute of Statistical Science, Taipei, 2002.

[15] X. Guo, Information and option pricings, Quantitative Finance 1 (2001), no. 1, 38-44.

[16] X. Guo and Q. Zhang, Closed-form solutions for perpetual American put options with regime switching, SIAM Journal on Applied Mathematics 64 (2004), no. 6, 2034-2049.

[17] _ Optimal selling rules in a regime switching model, IEEE Transactions on Automatic Control 50 (2005), no. 9, 1450-1455.

[18] R. W. Lee, Option pricing by transform methods: extensions, unification, and error control, Journal of Computational Finance 7 (2004), no. 3, 51-86.

[19] D. Madan, P. Carr, and E. Chang, The variance gamma process and option pricing, European Finance Review 2 (1998), 79-105. 
[20] M. Pemy and Q. Zhang, Optimal stock liquidation in a regime switching model with finite time horizon, Journal of Mathematical Analysis and Applications 321 (2006), 537-552.

[21] D. D. Yao, Q. Zhang, and X. Y. Zhou, A regime-switching model for European options, Stochastic Processes, Optimization, and Control Theory Applications in Financial Engineering, Queueing Networks, and Manufacturing Systems (H. M. Yan, G. Yin, and Q. Zhang, eds.), Springer, New York, 2006, pp. 281-300.

[22] G. Yin, R. H. Liu, and Q. Zhang, Recursive algorithms for stock liquidation: a stochastic optimization approach, SIAM Journal on Optimization 13 (2002), no. 1, 240-263.

[23] G. Yin and Q. Zhang, Continuous-Time Markov Chains and Applications. A Singular Perturbation Approach, Applications of Mathematics, vol. 37, Springer, New York, 1998.

[24] _ Discrete-Time Markov Chains. Two-Time-Scale Methods and Applications, Applications of Mathematics, vol. 55, Springer, New York, 2005.

[25] Q. Zhang, Stock trading: an optimal selling rule, SIAM Journal on Control and Optimization 40 (2001), no. 1, 64-87.

[26] Q. Zhang and G. Yin, Nearly-optimal asset allocation in hybrid stock investment models, Journal of Optimization Theory and Applications 121 (2004), no. 2, 419-444.

[27] Q. Zhang, G. Yin, and R. H. Liu, A near-optimal selling rule for a two-time-scale market model, SIAM Journal on Multiscale Modeling \& Simulation 4 (2005), no. 1, 172-193.

[28] X. Y. Zhou and G. Yin, Markowitz's mean-variance portfolio selection with regime switching: a continuous-time model, SIAM Journal on Control and Optimization 42 (2003), no. 4, 14661482.

R. H. Liu: Department of Mathematics, University of Dayton, 300 College Park, Dayton, OH 45469-2316, USA

E-mail address: ruihua.liu@notes.udayton.edu

Q. Zhang: Department of Mathematics, The University of Georgia, Athens, GA 30602-7403, USA

E-mail address: qingz@math.uga.edu

G. Yin: Department of Mathematics, Wayne State University, Detroit, MI 48202, USA

E-mail address: gyin@math.wayne.edu 


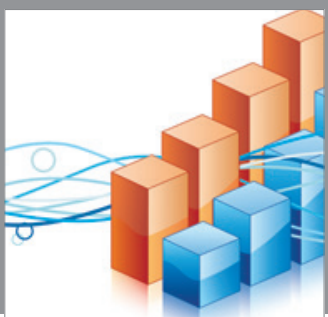

Advances in

Operations Research

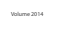

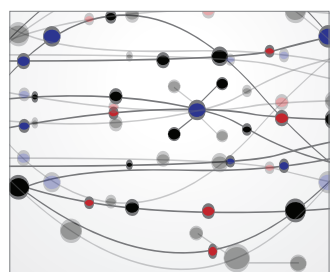

\section{The Scientific} World Journal
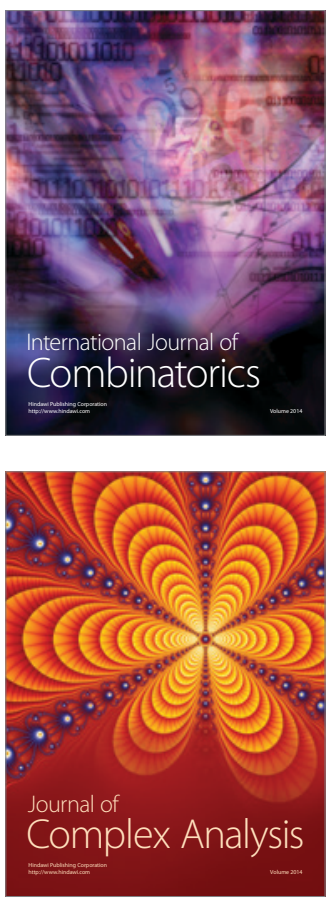

International Journal of

Mathematics and

Mathematical

Sciences
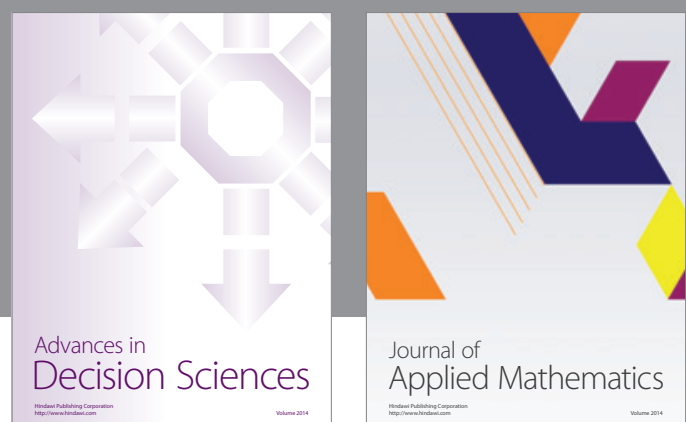

Journal of

Applied Mathematics
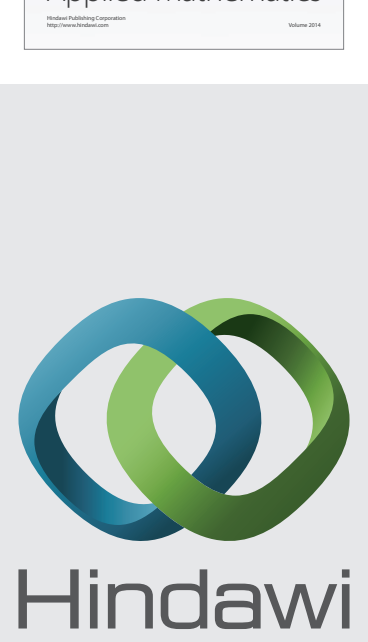

Submit your manuscripts at http://www.hindawi.com
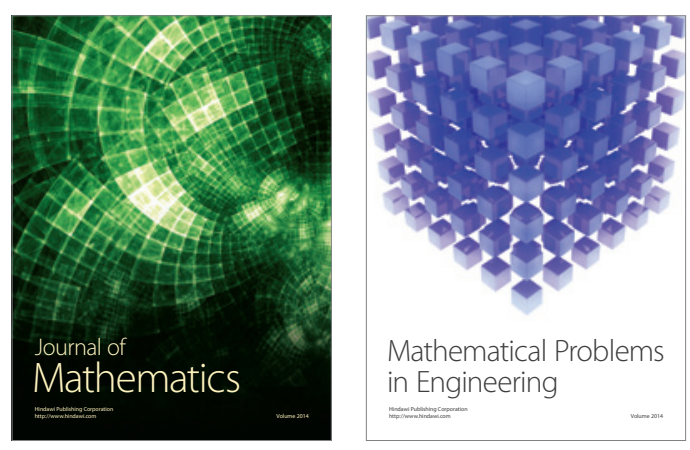

Mathematical Problems in Engineering
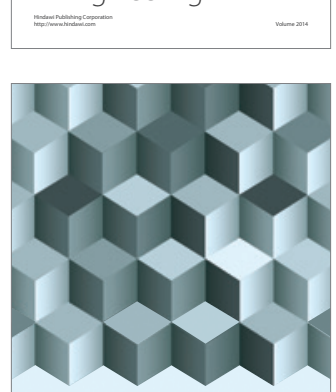

Journal of

Function Spaces
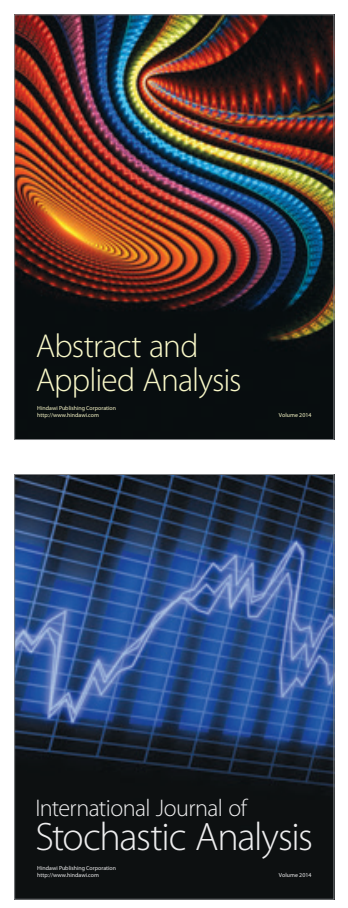

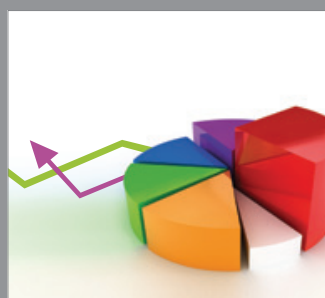

ournal of

Probability and Statistics

Promensencen
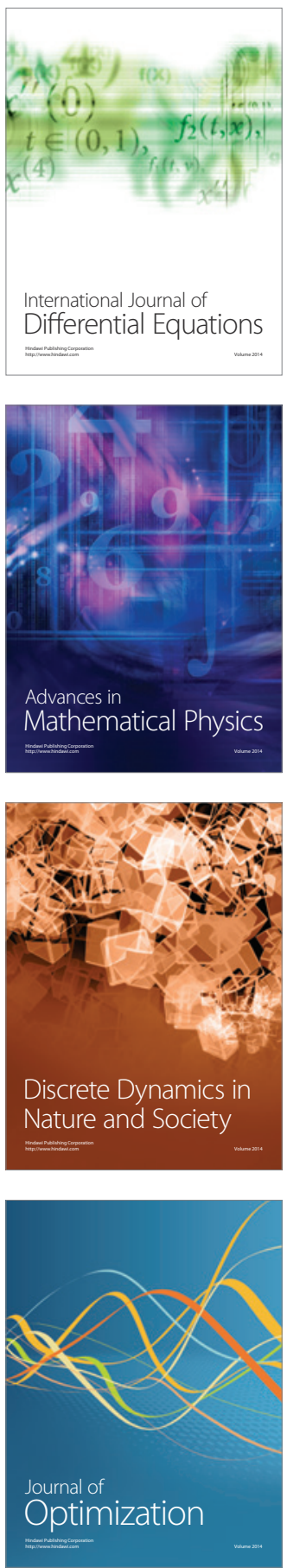